\title{
Evaluation of the Benefit of Radiotherapy in Patients with Occult Breast Cancer: A Population-Based Analysis of the SEER Database
}

\author{
Byoung Hyuck Kim, MD \\ Jeanny Kwon, MD, PhD² \\ Kyubo Kim, MD, $\mathrm{PhD}^{3}$
}

\begin{abstract}
Purpose
Few studies for occult breast cancer (OBC) have evaluated the effect of radiotherapy (RT) after mastectomy or axillary lymph node dissection (ALND) with/without breast surgery. Therefore, we investigated clinicopathologic factors of $\mathrm{OBC}$ with the impact of postoperative RT to determine its prognostic significance using large population-based data.
\end{abstract}

\section{Materials and Methods}

We performed a retrospective cohort study using the Surveillance, Epidemiology, and End Results (SEER) database from 1983 to 2013. A total of 1,045 eligible patients with OBC were identified. We compared overall survival (OS) using Cox proportional hazards regression with propensity score matching after verifying an imbalance of prognosticators between RT group ( $\mathrm{n}=518)$ and non-RT group $(\mathrm{n}=479)$.

\section{Results}

Patients with age $<70(p=0.033)$, married marital status $(p<0.001)$, undergoing ALND $(p<0.001)$, more examined lymph nodes (LNs) $(p<0.001)$, and more metastatic LNs $(p<0.001)$ were more likely to receive RT. Multivariate analysis after propensity score matching ( $n=798$ ) showed that patients treated with RT survived significantly longer than those without RT (5-year OS, $81.5 \%$ vs. $78.3 \%$; $p=0.014$ ). A significantly prolonged OS was observed when RT was given to patients treated with mastectomy $(p=0.033)$, those treated with ALND ( $p=0.036)$, or those with more than seven metastatic $L N s(p=0.016)$.

\section{Conclusion}

RT may offer survival benefit in OBC even after mastectomy or ALND, especially in patients with more than seven metastatic LNs. Further prospective studies are needed to validate these findings.
Co-correspondence: Kyubo Kim, MD, PhD Department of Radiation Oncology,

Ewha Womans University College of Medicine, 1071 Anyangcheon-ro, Yangcheon-gu,

Seoul 07985, Korea

Tel: $82-2-2650-5334$

Fax: 82-2-2654-0363

E-mail: kyubokim.ro@gmail.com

Received April 18, 2017

Accepted May 28, 2017

Published Online June 1, 2017
Key words

Occult breast cancer, Radiotherapy, SEER program

\section{Introduction}

Occult breast cancer (OBC) is a rare type of breast cancer which presents with axillary lymph node (LN) metastasis without identified primary breast lesion [1,2]. Current National Comprehensive Cancer Network (NCCN) guidelines recommend that axillary LN as well as ipsilateral breast should be treated with modified radical mastectomy or axil- lary LN dissection (ALND) plus whole breast radiotherapy (RT) even with no detectable breast tumor [3]. However, without prospective randomized trials due to its rarity, evidence on the managements of $\mathrm{OBC}$ comes from several retrospective studies including small number of patients [4-7].

Although systemic chemotherapy and endocrine therapy are believed to be performed according to the recommendations for their relatively comparative stage, locoregional managements for OBC such as breast conserving surgery 
(BCS), mastectomy, ALND, or its combinations have been variously implemented $[8,9]$. Previous studies suggested that BCS or ALND plus RT resulted in similar recurrence and survival rates compared to mastectomy plus ALND [5-7]. The first population-based data also demonstrated that both mastectomy plus ALND with/without postmastectomy RT and BCS with ALND and RT showed better survival than ALND alone but no difference between the formers was found [2]. However, from the standpoint of the radiation oncologist, no studies have evaluated the effect of RT after mastectomy or ALND with/without breast surgery. In addition, high-risk patients who need more aggressive locoregional treatments are not well-defined in the literature. Therefore, we investigated clinicopathologic factors of OBC with the impact of postoperative RT to determine its prognostic significance using large population-based data.

\section{Materials and Methods}

\section{Data source and study cohort}

We performed a retrospective population-based cohort study using the Surveillance, Epidemiology, and End Results (SEER) program of the National Cancer Institute in the United States. For present study, we identified 1,411 patients who were diagnosed with $\mathrm{OBC}(\mathrm{T} 0, \mathrm{~N}+)$ between January 1 , 1983 to December 31, 2013 in SEER 18 registry research data (Nov 2015 submission) using SEER *STAT software ver. 8.3.2. Among these patients, those who presented with distant metastasis ( $n=267)$ and those who were diagnosed with other malignancy within 2 years after diagnosis of OBC $(n=99)$ were excluded.

\section{Variables}

We extracted data on age at diagnosis, race, marital status, year of diagnosis, tumor grade, laterality, extent of breast operation, number of examined LNs, number of metastatic LNs, use of RT, status of estrogen receptor (ER) and progesterone receptor (PR) expression, and overall survival (OS). Race and marital status were simplified to three (white, black, or other) and two (single or married) categories, respectively. Extent of breast operation was classified into three categories including mastectomy, lumpectomy and no operation, according to the site specific surgery (1973-1997) and Rx summ-Surg Prim Site (after 1998) code. An extent of regional $\mathrm{LN}$ dissection was not specified in most cases in the database. Thus, we regarded the patients who underwent extended mastectomy, modified radical mastectomy, or dis- section of four or more regional LNs as having taken ALND according to the previous study of Walker et al. [2] which also used SEER database from 1983 to 2006. Variables included in subsequent analysis were outlined in Table 1.

\section{Propensity score matching with multiple imputation}

Because RT was not randomly assigned in our dataset, multivariate model using Cox regression analysis alone may not fully adjust many confounding factors and possible selection bias. To evaluate the impact of RT more accurately, we first compared the distribution of clinicopathologic factors between RT group and non-RT group; $\mathrm{t}$ test for continuous variables and chi-square test for categorical variables were used. After verifying an imbalance of prognosticators between two groups, we conducted a propensity score matching. However, there were too many missing values in the expression status of hormone receptor to match patients each other. Therefore, multiple imputation methods as in the study of Sagara et al. [10] were applied by MICE package in $\mathrm{R}$ with the following variables: age at diagnosis (as continuous variable), race, marital status, number of examined LNs, number of metastatic LNs and extent of operation. The imputation was repeated to obtain five copies of the filledin data set to stabilize the results.

After imputing missing values, propensity score for RT receipt was calculated for each patient using multivariate logistic regression, considering all of the imbalanced factors between RT and non-RT group: age at diagnosis (as continuous variable), marital status, ALND, number of examined LNs, and number of metastatic LNs. Based on the results, we performed propensity score matching with a 1:1 nearestneighbor method. Distribution of propensity scores was depicted in S1 Fig.

\section{Endpoint and statistical analysis}

OS was defined as the duration from the date of diagnosis to the date of last follow-up or death from any cause. For survival analysis, Kaplan-Meier method was used to estimate survival curve and log-rank test was performed for the comparison of survival between the nominal variables. Cut-off values for the number of examined or metastatic LNs were determined by maximal chi-square test. All variables that were significant in univariate analysis were put into multivariate analysis. Multivariate analysis was carried through Cox proportional hazard regression. Factors with $\mathrm{p}>0.10$ were eliminated from the stepwise model. All statistical analyses were conducted using R software ver. 3.2.4 (http:// cran.r-project.org). p-value of less than 0.05 was considered statistically significant. 
Table 1. Patient and tumor characteristics

\begin{tabular}{|c|c|}
\hline Characteristic & $\begin{array}{l}\text { No. of patients }(\%) \\
(n=1,045)\end{array}$ \\
\hline \multicolumn{2}{|l|}{ Age at diagnosis (yr) } \\
\hline Median (IQR) & $59(51-69)$ \\
\hline$<40$ & $54(5.2)$ \\
\hline $40-69$ & $751(71.9)$ \\
\hline$\geq 70$ & $240(23.0)$ \\
\hline \multicolumn{2}{|l|}{ Race } \\
\hline White & $830(79.4)$ \\
\hline Black & $133(12.7)$ \\
\hline Others & $78(7.5)$ \\
\hline Unknown & $4(0.4)$ \\
\hline \multicolumn{2}{|l|}{ Marital status } \\
\hline Single & $408(39.0)$ \\
\hline Married & $607(58.1)$ \\
\hline Unknown & $30(2.9)$ \\
\hline \multicolumn{2}{|l|}{ Year of diagnosis } \\
\hline 1988-1997 & $85(8.1)$ \\
\hline $1998-2003$ & $169(16.2)$ \\
\hline 2004-2008 & $376(36.0)$ \\
\hline $2009-2013$ & $415(39.7)$ \\
\hline \multicolumn{2}{|l|}{ Grade } \\
\hline 1 & $10(0.1)$ \\
\hline 2 & $54(5.2)$ \\
\hline 3 & $235(22.5)$ \\
\hline Undifferentiated & $21(2.0)$ \\
\hline Unknown & $725(69.4)$ \\
\hline \multicolumn{2}{|l|}{ Laterality } \\
\hline Right & $475(45.5)$ \\
\hline Left & $518(49.6)$ \\
\hline Bilateral & $3(0.3)$ \\
\hline Unknown & $49(4.7)$ \\
\hline \multicolumn{2}{|c|}{ Estrogen receptor status } \\
\hline Negative & $300(28.7)$ \\
\hline Positive & $406(38.9)$ \\
\hline Unknown & $339(32.4)$ \\
\hline \multicolumn{2}{|c|}{ Progesterone receptor status } \\
\hline Negative & $410(39.2)$ \\
\hline Positive & $283(27.1)$ \\
\hline Unknown & $352(33.7)$ \\
\hline \multicolumn{2}{|l|}{ Breast operation } \\
\hline Mastectomy & $411(39.3)$ \\
\hline Lumpectomy & $140(13.4)$ \\
\hline Not done & $477(45.7)$ \\
\hline Unknown & $17(1.6)$ \\
\hline \multicolumn{2}{|l|}{ Axilla LN operation } \\
\hline ALND & $805(77.0)$ \\
\hline ALNS $(\leq 3)$ & $237(22.7)$ \\
\hline Unknown & $3(0.3)$ \\
\hline
\end{tabular}

(Continued)
Table 1. Continued

\begin{tabular}{|lc} 
Characteristic & $\begin{array}{c}\text { No. of patients }(\%) \\
(\mathbf{n}=\mathbf{1}, \mathbf{0 4 5})\end{array}$ \\
\hline $\begin{array}{l}\text { Extent of operation } \\
\text { Mastectomy+ALND }\end{array}$ & $318(30.4)$ \\
Mastectomy alone & $92(8.8)$ \\
\hline Lumpectomy+ALND & $114(10.9)$ \\
\hline Lumpectomy alone & $26(2.5)$ \\
\hline ALND alone & $358(34.2)$ \\
\hline ALNS alone & $117(11.2)$ \\
\hline Unknown & $20(1.9)$ \\
\hline Radiotherapy & $479(45.8)$ \\
\hline Not done & $518(49.6)$ \\
\hline Done & $48(4.6)$ \\
\hline Unknown & $13(6-20)$ \\
\hline No. of examined LNs & \\
\hline Median (IQR) & $2(1-6)$ \\
\hline No. of metastatic LNs & \\
\hline Median (IQR) & \\
\hline
\end{tabular}

$\mathrm{IQR}$, interquartile range; $\mathrm{LN}$, lymph node; ALND, axillary lymph node dissection; ALNS, axillary lymph node sampling.

\section{Results}

\section{Patient characteristics and treatment patterns}

A total of 1,045 patients were included in the final analysis. Patient demographics were presented in Table 1 . The median age was 59 years (interquartile range [IQR], 51 to 69). Mastectomy was carried out in 411 patients $(39.3 \%)$ and ALND in 805 patients $(77.0 \%)$. RT was delivered to nearly half of the patients $(49.6 \%)$. The median number of examined and metastatic LNs were 13 (IQR, 6 to 20) and 2 (range, 1 to 6), respectively. In terms of locoregional treatment, various combinations of surgical extent and RT were observed (S2 Table). Around $23 \%$ of patients underwent breast operation with ALND followed by RT (171 patients with mastectomy+ ALND+RT, 69 patients with BCS+ALND+RT). In contrast, 76 patients $(7.3 \%)$ did not receive any treatment including breast operation or RT, but underwent only axillary LN sampling (ALNS) or excision for less than four LNs.

\section{Survival analysis in whole SEER cohort}

The median follow-up duration was 56 months (IQR, 24 to 100). During follow-up period, 257 deaths (24.6\%) from any cause occurred and 5-year OS was 79.1\%. Results of survival analyses were summarized in Table 2 . Univariate analysis 
Table 2. Univariate and multivariate analyses for OS in the study population

\begin{tabular}{|c|c|c|c|c|}
\hline Characteristic & $\begin{array}{l}\text { 5-Year } \\
\text { OS }(\%)\end{array}$ & $\begin{array}{l}\text { Univariate } \\
\text { analysis }^{a}\end{array}$ & $\begin{array}{l}\text { Multivariate } \\
\text { analysis }^{\text {b) }}\end{array}$ & HR $(95 \%$ CI) \\
\hline \multicolumn{5}{|l|}{ Age at diagnosis (yr) } \\
\hline$<70$ & 83.5 & $<0.001$ & $<0.001$ & 1 \\
\hline$\geq 70$ & 63.0 & & & $2.71(1.67-4.39)$ \\
\hline \multicolumn{5}{|l|}{ Marital status } \\
\hline Single & 73.8 & $<0.001$ & - & - \\
\hline Married & 83.3 & & & - \\
\hline \multicolumn{5}{|l|}{ Grade } \\
\hline 1 & 88.9 & 0.900 & - & - \\
\hline 2 & 83.5 & & & - \\
\hline 3 & 77.3 & & & - \\
\hline Undifferentiated & 79.8 & & & - \\
\hline \multicolumn{5}{|l|}{ ER expression } \\
\hline Negative & 76.9 & 0.025 & 0.064 & 1 \\
\hline Positive & 83.1 & & & $0.65(0.42-1.02)$ \\
\hline \multicolumn{5}{|l|}{ PR expression } \\
\hline Negative & 77.5 & 0.049 & - & - \\
\hline Positive & 84.1 & & & - \\
\hline \multicolumn{5}{|l|}{ Breast operation } \\
\hline Mastectomy & 74.6 & 0.123 & - & - \\
\hline Lumpectomy & 80.3 & & & - \\
\hline Not done & 79.1 & & & - \\
\hline \multicolumn{5}{|l|}{ Axilla LN operation } \\
\hline ALNS $(\leq 3)$ & 68.9 & $<0.001$ & 0.003 & 1 \\
\hline ALND & 81.7 & & & $0.42(0.24-0.74)$ \\
\hline \multicolumn{5}{|l|}{ Radiotherapy } \\
\hline Not done & 74.8 & 0.001 & 0.010 & 1 \\
\hline Done & 82.1 & & & $0.53(0.33-0.86)$ \\
\hline \multicolumn{5}{|l|}{ No. of examined LNs } \\
\hline$<3$ & 72.8 & $<0.001$ & - & - \\
\hline$\geq 3$ & 84.1 & & & - \\
\hline \multicolumn{5}{|c|}{ No. of metastatic LNs } \\
\hline$\leq 7$ & 85.6 & $<0.001$ & $<0.001$ & 1 \\
\hline$>7$ & 70.2 & & & $2.81(1.65-4.80)$ \\
\hline
\end{tabular}

OS, overall survival; HR, hazard ratio; CI, confidence interval; ER, estrogen receptor; PR, progesterone receptor; LN, lymph node; ALNS, axillary lymph node sampling; ALND, axillary lymph node dissection. ${ }^{a}$ p-value by log-rank test, b)p-value by Cox proportional hazard model with backward stepwise regression.

revealed that age more than 70 years $(\mathrm{p}<0.001)$, single marital status $(\mathrm{p}<0.001)$, absence of hormone receptor expression (ER, $\mathrm{p}=0.025 ; \mathrm{PR}, \mathrm{p}=0.049)$, not performing ALND $(\mathrm{p}<0.001)$ or not doing RT $(\mathrm{p}=0.001)$, number of examined LNs less than three $(p<0.001)$, and metastatic LNs more than seven $(\mathrm{p}<0.001)$ were poor prognosticators for OS. Survival difference according to the surgical extent of breast was not observed $(\mathrm{p}=0.123)$ (Table 2).

Multivariate analysis was performed including abovementioned factors. After adjustment of confounding factors, age more than 70 years (hazard ratio [HR], 2.71; $\mathrm{p}<0.001$ ), performing ALND (HR, 0.42; $\mathrm{p}=0.003$ ) or doing RT (HR, 0.53; $\mathrm{p}=0.010$ ), and metastasis to more than seven LNs (HR, 2.81; $\mathrm{p}<0.001)$ were independently significant prognostic variables. The OS curves according to the significant prognosticators were demonstrated in S3 Fig. 


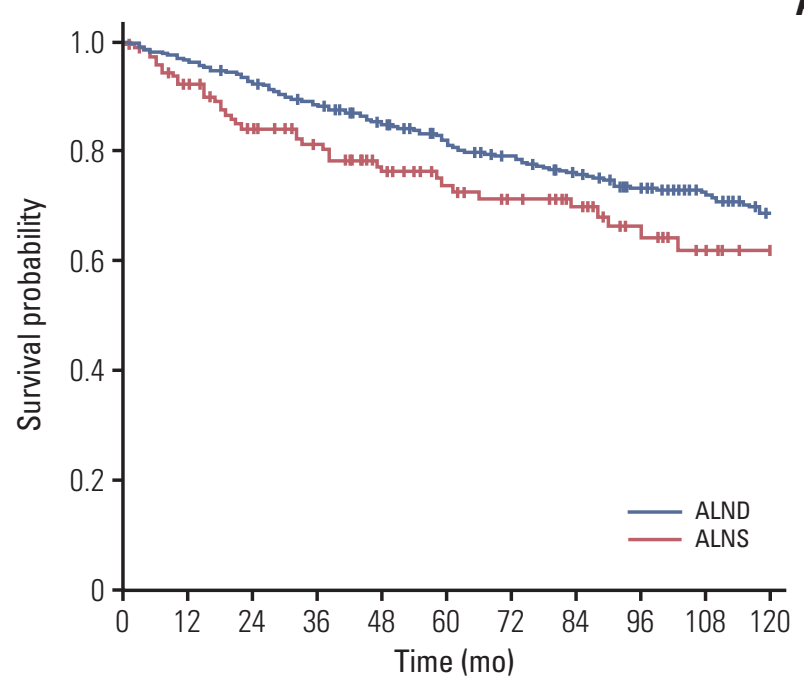

A

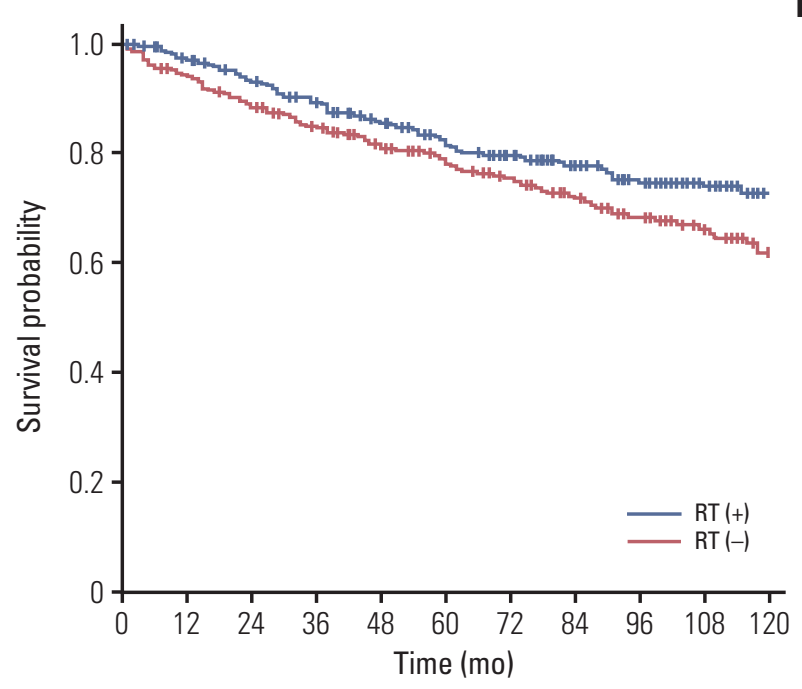

C

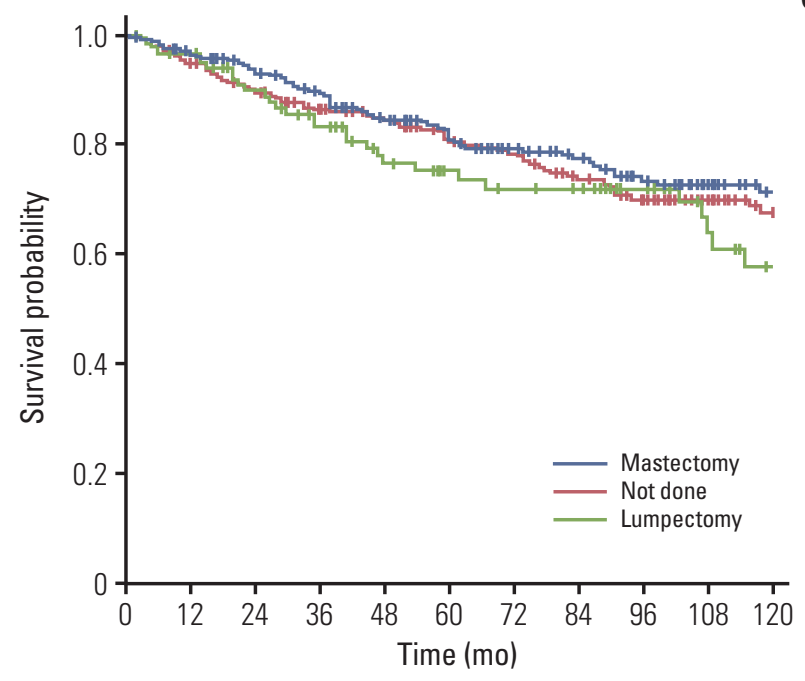

Fig. 1. Kaplan-Meier curve of overall survival in propensity score matched cohort according to the axillary lymph node dissection (ALND) (A), radiotherapy (RT) (B), and type of breast operation (C). ALNS, axillary lymph node sampling.

\section{Survival analysis in propensity score matched cohort}

We compared patient characteristics according to the receipt of RT (S4 Table). Prior to matching, young patients $(\mathrm{p}=0.033)$, patients with married marital status $(\mathrm{p}<0.001)$, patient treated with ALND $(\mathrm{p}<0.001)$, patients with more examined LNs $(p<0.001)$ and patients with more metastatic LNs $(p<0.001)$ were more likely to receive RT. The observed imbalances between two groups were corrected through propensity score matching, as a result, none of the variables was significantly different between two groups as presented in S4 Table. 
Table 3. Univariate and multivariate analyses for OS in propensity score matched cohort

\begin{tabular}{|c|c|c|c|c|}
\hline Characteristic & $\begin{array}{l}\text { 5-Year } \\
\text { OS }(\%)\end{array}$ & $\begin{array}{l}\text { Univariate } \\
\text { analysis }^{a}\end{array}$ & $\begin{array}{l}\text { Multivariate } \\
\text { analysis }^{\text {b) }}\end{array}$ & HR $(95 \%$ CI) \\
\hline \multicolumn{5}{|l|}{ Age at diagnosis (yr) } \\
\hline$<70$ & 84.0 & $<0.001$ & $<0.001$ & 1 \\
\hline$\geq 70$ & 64.1 & & & $2.81(1.95-4.03)$ \\
\hline \multicolumn{5}{|l|}{ Marital status } \\
\hline Single & 75.1 & $<0.001$ & 0.047 & 1 \\
\hline Married & 83.7 & & & $0.70(0.50-0.10)$ \\
\hline \multicolumn{5}{|l|}{ Grade } \\
\hline 1 & 100 & 0.945 & - & - \\
\hline 2 & 81.3 & & & - \\
\hline 3 & 77.2 & & & - \\
\hline Undifferentiated & 79.6 & & & - \\
\hline \multicolumn{5}{|l|}{ ER expression } \\
\hline Negative & 77.6 & 0.295 & - & - \\
\hline Positive & 81.6 & & & - \\
\hline \multicolumn{5}{|l|}{ PR expression } \\
\hline Negative & 78.9 & 0.778 & - & - \\
\hline Positive & 81.4 & & & - \\
\hline \multicolumn{5}{|l|}{ Breast operation } \\
\hline Mastectomy & 75.4 & 0.136 & - & - \\
\hline Lumpectomy & 80.2 & & & - \\
\hline Not done & 81.3 & & & - \\
\hline \multicolumn{5}{|l|}{ Axilla LN operation } \\
\hline ALNS $(\leq 3)$ & 74.3 & 0.011 & $<0.001$ & 1 \\
\hline ALND & 81.1 & & & $0.44(0.28-0.68)$ \\
\hline \multicolumn{5}{|l|}{ Radiotherapy } \\
\hline Not done & 78.3 & 0.049 & 0.014 & 1 \\
\hline Done & 81.5 & & & $0.65(0.46-0.92)$ \\
\hline \multicolumn{5}{|l|}{ No. of examined LNs } \\
\hline$<3$ & 78.6 & 0.057 & - & - \\
\hline$\geq 3$ & 82.8 & & & - \\
\hline \multicolumn{5}{|c|}{ No. of metastatic LNs } \\
\hline$\leq 7$ & 86.5 & $<0.001$ & $<0.001$ & 1 \\
\hline$>7$ & 66.2 & & & $3.09(2.10-4.53)$ \\
\hline
\end{tabular}

OS, overall survival; HR, hazard ratio; CI, confidence interval; ER, estrogen receptor; PR, progesterone receptor; LN, lymph node; ALNS, axillary lymph node sampling; ALND, axillary lymph node dissection. ${ }^{a}$ p-value by log-rank test, b)p-value by Cox proportional hazard model with backward stepwise regression.

sis in propensity matched cohort were summarized in Table 3.

\section{Exploratory subgroup analysis assessing the benefit of RT according to the characteristics}

To identify a specific subgroup which could benefit from $\mathrm{RT}$, we conducted exploratory subgroup analysis in propensity score matched cohort. Overall, Fig. 2 plotted HR and 95\% confidence interval comparing OS according to the receipt of RT for each patient characteristic. Patients responded differently to RT depending upon several factors. A significantly prolonged OS was observed when RT was given to patients single marital status (5-year OS, $81.0 \%$ vs. $69.5 \%$; $\mathrm{p}=0.001$ ), patients treated with mastectomy (5-year OS, $84.9 \%$ vs. 77.4\%; $\mathrm{p}=0.033$ ) (Fig. 3A), patients treated with ALND (5-year OS, $83.3 \%$ vs. $78.8 \%$; $\mathrm{p}=0.036$ ) (Fig. 3B) or patients with more than seven metastatic LNs (5-year OS, $70.7 \%$ vs. $59.7 \% ; \mathrm{p}=0.016)$. A trend toward improved OS by adding RT was also detected in patients without expression of hormone receptor (ER, 5-year OS, $79.7 \%$ vs. $75.2 \%$; $\mathrm{p}=0.070$ and $\mathrm{PR}$, 5 -year OS, $80.7 \%$ vs. $76.9 \%$; $\mathrm{p}=0.053)$. 


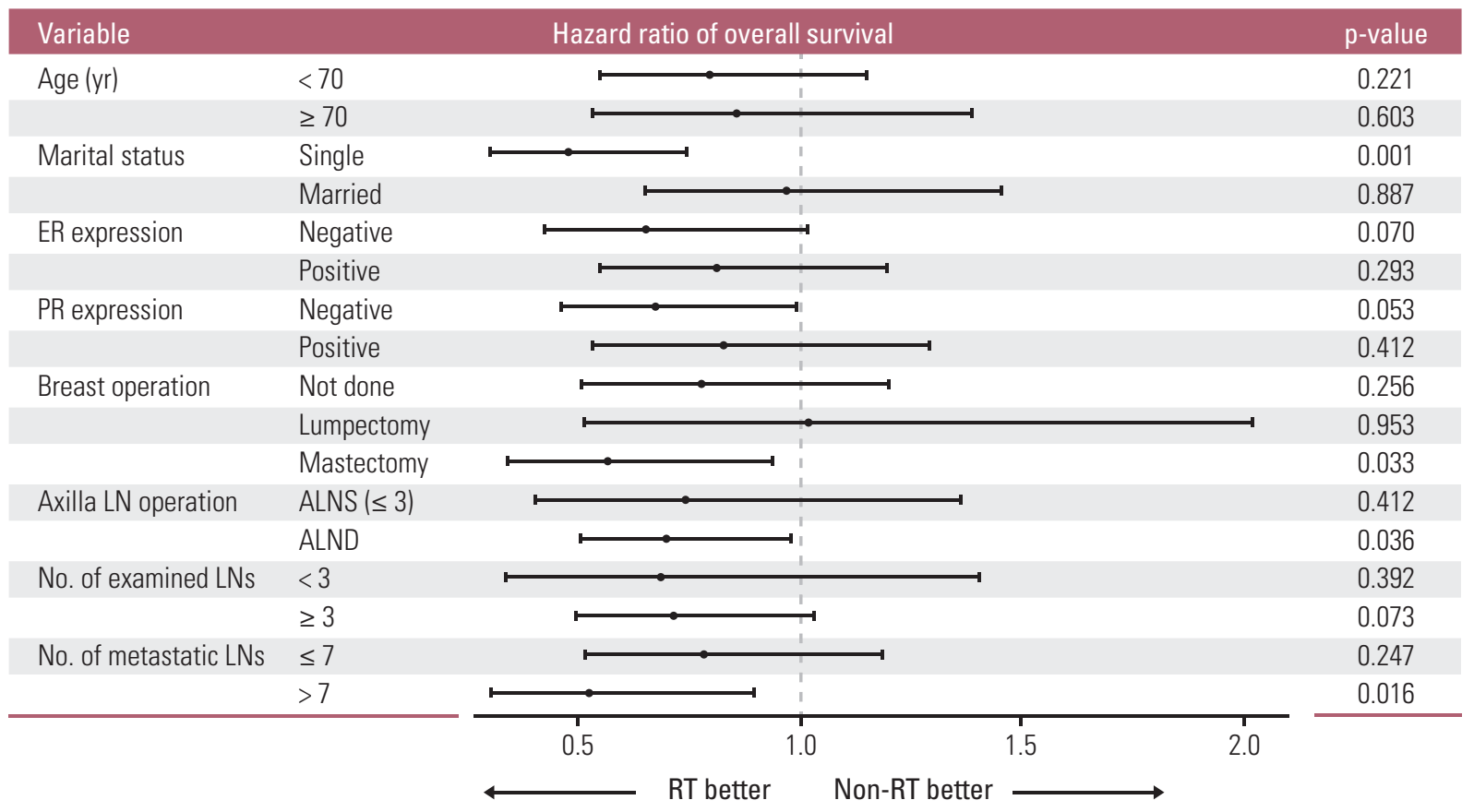

Fig. 2. Hazard ratio and $95 \%$ confidence interval for overall survival according to the receipt of radiotherapy (RT) in the subgroup of patients for each characteristic. ER, estrogen receptor; PR, progesterone receptor; LN, lymph node; ALNS, axillary lymph node sampling; ALND, axillary lymph node dissection.

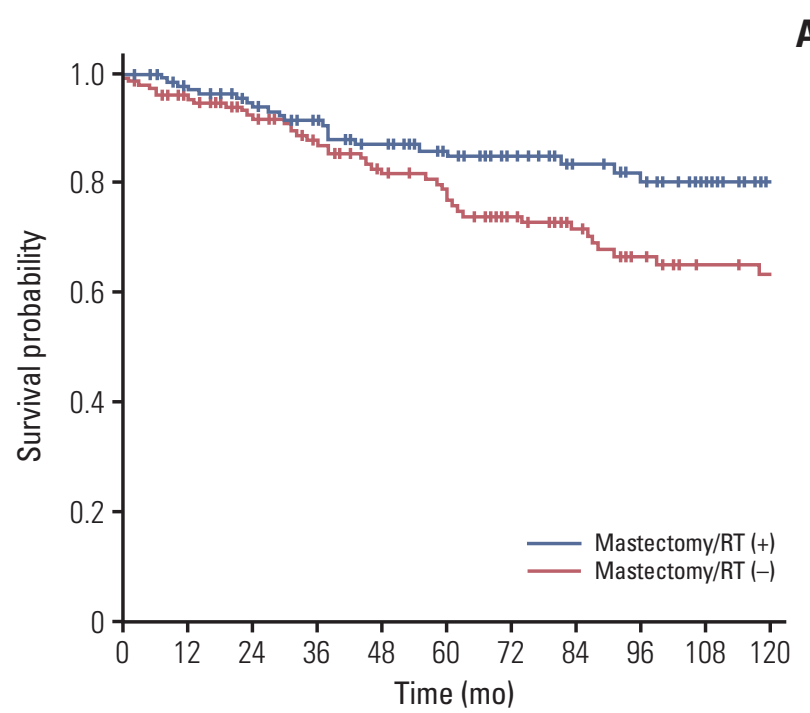

A

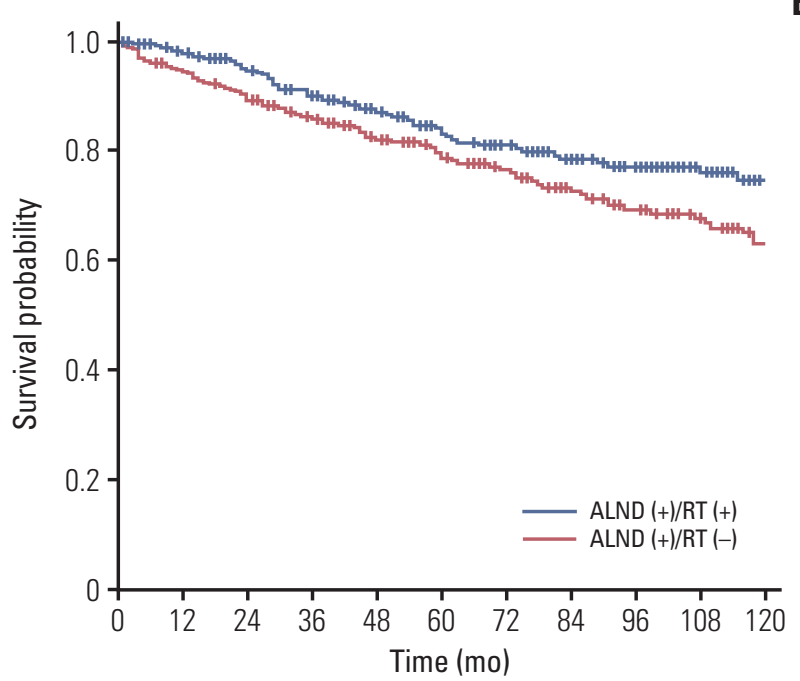

Fig. 3. Kaplan-Meier curve of overall survival in propensity score matched cohort according to the receipt of radiotherapy (RT) in the subgroup of patients treated with mastectomy (A) and axillary lymph node dissection (ALND) (B). 


\section{Discussion}

The main purpose of this population-based retrospective study was to evaluate the clinicopathologic factors affecting OS as well as the impact of RT under different surgical conditions for OBC. Although ALND followed by breast RT is accepted as one of the preferred strategy after many comparisons with mastectomy have been made, the impact of RT after ALND or mastectomy compared with each surgical treatment alone has not been exactly evaluated in previous literatures [5-7]. It is hard to conduct a randomized trial because of the rarity, therefore we used retrospective matching analysis using large database. To the best of knowledge, present updated SEER analysis represents the largest cohort to date in which survival outcome for OBC was analyzed. Significant improvements in survival in the RT group compared with the non-RT group were generally observed, also specifically in patients with single marital status, receiving mastectomy, receiving ALND, and number of metastatic LNs more than 7 .

Treatment patterns varied over the years. Although mastectomy did not improve OS compared with breast-conserving therapy (BCT), there were quite a large proportion of patients treated with mastectomy. Previous survey for the members of the American Society of Breast Surgeons demonstrated that mastectomy with ALND preferred in 43\%, whereas ALND with whole breast RT in 37\% [9]. A previous study for OBC using the SEER data (1983-2006) demonstrated that number of patients who received BCT increased from $29.8 \%$ before 1998 to $36.2 \%$ after 1998 , whereas number of patients who received mastectomy decreased from 50.2\% to $42 \%$ during the same period, which still held a significant portion [2]. Although BCT is replacing mastectomy with the advent of diagnostic imaging, mastectomy still have been performed in many patients due to physician's preference or clinical factors, but the role of RT in these patients was also not separately discussed in previous studies $[11,12]$. Our analysis revealed that RT after mastectomy was an effective adjuvant treatment to reduce mortality, as with survival benefit by adding RT in other node positive breast cancers [13-15]. This survival benefit might derive from the comprehensive nodal irradiation, although the detailed RT field is unavailable from the SEER database. Also, the NCCN guidelines do not mention this in detail. Therefore, physicians should discuss with radiation oncologists to determine whether postmastectomy RT is appropriate or not, even if subsequent validation studies will be required

The standard recommendations in terms of RT field and dose are not well defined in this rare disease. By reviewing the contemporary studies using RT, Table 4 shows that most of RT field includes ipsilateral breast in case of BCS, whereas there is not much information about chest wall irradiation after mastectomy. Among them, Barton et al. [16] recommended the incorporation of ipsilateral breast RT due to the higher 5-year local recurrence-free survival ( $84 \%$ vs. $34 \%$, $\mathrm{p}<0.001)$ and relapse-free survival (64\% vs. $34 \%, \mathrm{p}=0.05)$. The extent or frequency of regional nodal irradiation is also varied for each study, so it is difficult to summarize the conclusions. However, more than $70 \%$ of patients received some kinds of regional nodal irradiation, mainly including supraclavicular fossa. In terms of RT dose, a total dose of 40 to 60 Gy has been applied variously according to the surgical extents and residual disease. A previous study showed that no difference in the 5-year local recurrence-free survival $(80 \%$ vs. $90 \%, p=0.3$ ) was found between patients who received 50 Gy versus more than 60 Gy [16]. The present study could not address these aspects because information about the exact delivery site of RT (whole breast irradiation, localized axillary RT, or regional nodal irradiation) and RT dose was not included in SEER data. Further specific guidelines will be needed.

The benefit of adding RT is not limited just after mastectomy. Previous studies have rather recommended incorporation of breast RT with ALND. Historically, locoregional recurrence was too high (around 50\%) in patients who did not receive breast treatment after ALND [17-19]. Shannon et al. [17] reported that $69 \%$ of patients without breast RT underwent locoregional recurrence; however, this figure decreased to $12.5 \%$ in those receiving breast $\mathrm{RT}$ ( $\mathrm{p}=0.02$ ). Vlastos et al. [12] also reported that patients who were observed without breast treatment showed $50 \%$ OS, whereas those who received breast RT after axillary surgery showed $83 \%$ OS. Therefore, "blind" treatment to the ipsilateral breast seemed to be important. Our results showed that application of RT has a positive effect on OS rate in both unmatched and matched cohort. Especially, patients treated with ALND also derived OS benefit from RT in subgroup analysis. A recent meta-analysis including seven previous studies demonstrated that ALND plus RT was superior to ALND alone regarding locoregional recurrence $(12.7 \%$ vs. $34.3 \%, \mathrm{p}<0.01)$ and there was a trend toward improved mortality rates $(9.5 \%$ vs. $31.4 \%, \mathrm{p}=0.09$ ) [20]. In addition, MD Anderson Cancer Center reported that there were no local or regional failures in $36 \mathrm{OBC}$ patients dealt with contemporary imaging and multimodal BCT [7]. These data supported our findings by indicating that breast RT after axillary treatment only is mandatory and ALND combined with RT also appears as an attractive option to avoid mastectomy and its complications. Therefore, mastectomy could be safely omitted in the setting where a patient is willing to undergo breast RT after ALND.

One of the interesting points of our results was that the extent of breast operation (mastectomy/lumpectomy/not done) did not affect OS significantly. Instead, ALND was an 


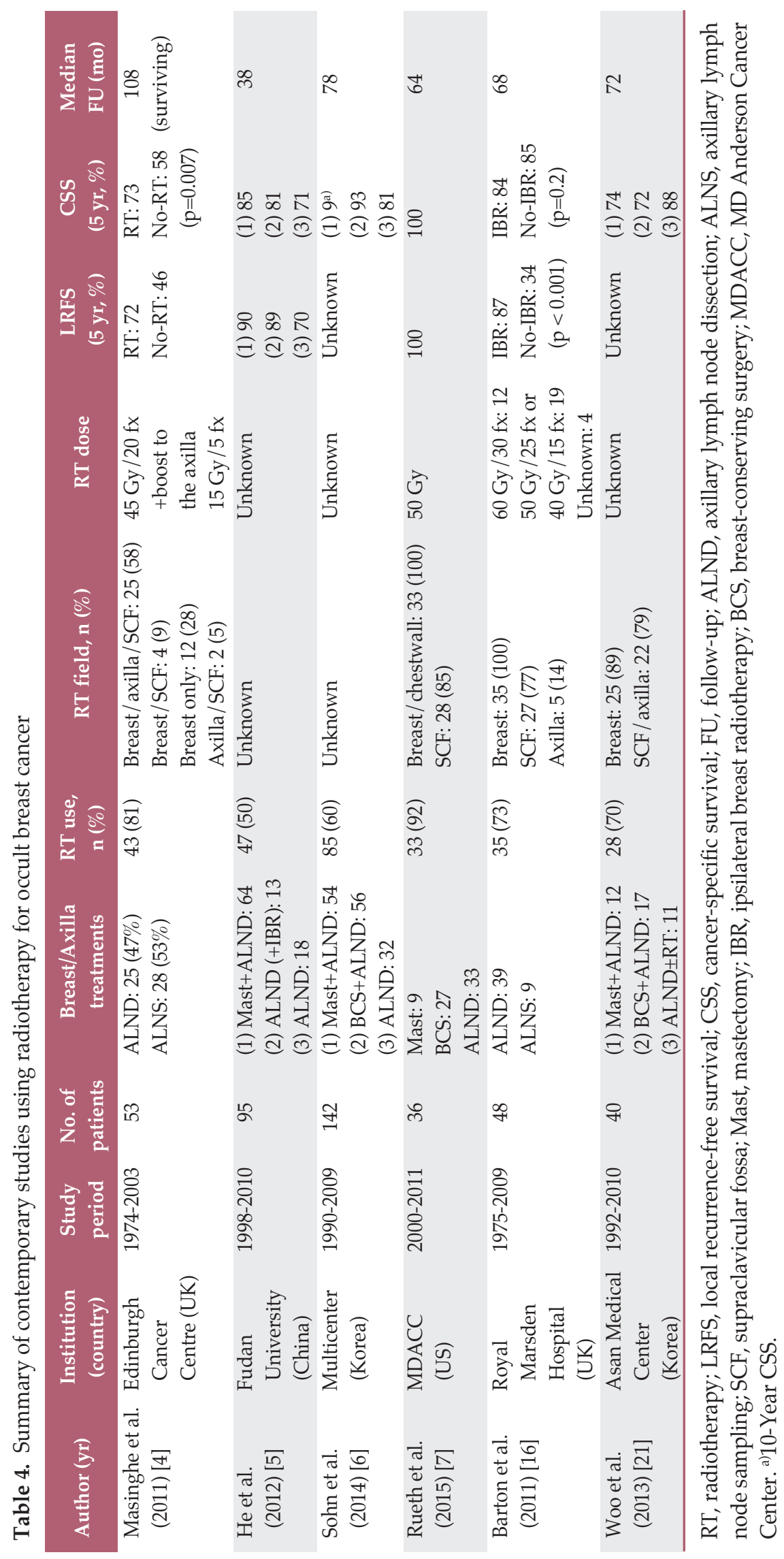


important prognostic factor. Previous studies also reported similar findings. The 5-year OS of BCS, mastectomy, and no operation groups was $72.0 \%, 74.0 \%$, and $87.5 \%$, respectively, with no significant differences observed between them $(p=0.49)$ in the study by Woo et al. [21]. He et al. [5] also demonstrated that there were no differences in survival rates between mastectomy, BCS, and no breast operation. This may be due to the nature of $\mathrm{OBC}$ that an axillary clearance rather than breast operation could further reduce overall tumor burden. However, this does not mean that the breast operation is not important. In our results, a significantly prolonged OS was observed when RT was given to patients treated with mastectomy or ALND, not patients with lumpectomy or ALNS (Fig. 2). It is possible that there was a survival benefit of RT in the situations of more radical surgery. Also, as with the previous literatures mentioned above, the "blind" breast RT after BCS is effective for local control, but this may not be translated into OS differences $[5,16]$. More precise data are needed to clarify the impact of breast operation or blind breast RT on OS.

Regardless of the surgical approach, one of the most important prognostic factors was the number of positive nodes. Five-year OS was $85.6 \%$ with $1-7$ positive nodes, whereas only $70.2 \%$ with eight or more positive nodes. This tendency was maintained regardless of propensity score matching. Walker et al. [2] reported that more than 10 positive LNs were correlated with an unfavorable OS (HR, 2.04; $p=0.005)$. He et al. [5] also showed that patients with four or more involved LNs had significantly worse outcomes (HR, 4.63; $\mathrm{p}=0.042$ for survival and HR, 3.62; $\mathrm{p}=0.038$ for recurrence). Recent multicenter study from Korea demonstrated that patients who had N1 disease showed a fairly good 10-year OS of $96.8 \%$ compared with $82.6 \%$ in $\mathrm{N} 2$ and $80.8 \%$ in N3 disease $(\mathrm{p}=0.004)$ [6]. Therefore, adequate ALND should be performed to identify accurate LN status and also reduce the chance of occult nodal metastases. Additionally, age over 70 years at diagnosis was found to be a poor prognosticator in multivariate analysis. Similar results have been reported in the previous SEER data (RR, 1.06 with age as a continuous variable; $p<0.001)$ [2]. It seems a little bit contradictory considering the poor prognostic impact of young age in common breast cancer. Because the endpoint of our study was OS but not breast cancer-specific survival, it is not surprising that inferior survival outcomes were observed in the elderly patients. However, unknown factors such as systemic treatment, intrinsic molecular subtype or histologic grade might have induced the above results. In other literatures on $\mathrm{OBC}$, no significant difference was reported according to the age-related parameters.

One of the major limitations of this study is an absence of information on systemic chemotherapy and endocrine therapy due to the limitation of SEER data, thus could not be included in the analysis. The lack of these important factors could make potential bias because of heterogeneous systemic treatments during a relatively long period with improvements in outcome over the recent period, although one previous review for the managements of $\mathrm{OBC}$ reported that most patients in United States (93\%) had received chemotherapy [8]. Current NCCN guidelines recommend that systemic chemotherapy/endocrine therapy be given according to the stage II or III disease as well as neoadjuvant therapy also be considered for N2-3 disease [3]. In addition, the effects of chemotherapy have not been reported to be particularly different in this orphan disease in relation to common breast cancer. These observations emphasize the need for additional large sample studies to better understand prognostic impacts of systemic chemotherapy/endocrine therapy for this rare disease. There are another several limitations to this study. Retrospective nature is an unavoidable weakness although we tried to obtain unbiased comparison by using propensity score matching. However, propensity score matching itself could not solve the problem of hidden bias caused by unexamined factors, and there were lots of missing values which were dealt with imputation methods. Last but not least, failure patterns could not be analyzed. It is important to point out that locoregional recurrence is not captured in SEER data, being limited to survival outcomes.

Based on our results, RT may offer survival benefit in OBC even after mastectomy or ALND, especially in patients with more metastatic LNs. Combined ALND and RT irrespective of breast operation may appear as the best treatment approach in these patients favoring minimal invasive procedure. Age and number of metastatic LNs were also significant prognosticators. Individualized locoregional treatment strategies based on certain risk factors needs to be further investigated. Prospective studies with a sufficient sample size are needed to validate these findings.

\section{Electronic Supplementary Material}

Supplementary materials are available at Cancer Research and Treatment website (http:// www.e-crt.org).

\section{Conflicts of Interest}

Conflict of interest relevant to this article was not reported. 


\section{References}

1. Patel J, Nemoto T, Rosner D, Dao TL, Pickren JW. Axillary lymph node metastasis from an occult breast cancer. Cancer. 1981;47:2923-7.

2. Walker GV, Smith GL, Perkins GH, Oh JL, Woodward W, Yu TK, et al. Population-based analysis of occult primary breast cancer with axillary lymph node metastasis. Cancer. 2010;116: 4000-6.

3. National Comprehensive Cancer Network. NCCN clinical practice guidelines in oncology, Breast cancer. v.2.2016 [Internet]. Fort Washington, PA: National Comprehensive Cancer Network; 2016 [cited 2017 Jun 1]. Available from: http:// www. nccn.org/professionals/physician_gls/pdf/breast.pdf.

4. Masinghe SP, Faluyi OO, Kerr GR, Kunkler IH. Breast radiotherapy for occult breast cancer with axillary nodal metastases: does it reduce the local recurrence rate and increase overall survival? Clin Oncol (R Coll Radiol). 2011;23:95-100.

5. He M, Tang LC, Yu KD, Cao AY, Shen ZZ, Shao ZM, et al. Treatment outcomes and unfavorable prognostic factors in patients with occult breast cancer. Eur J Surg Oncol. 2012;38: 1022-8.

6. Sohn G, Son BH, Lee SJ, Kang EY, Jung SH, Cho SH, et al. Treatment and survival of patients with occult breast cancer with axillary lymph node metastasis: a nationwide retrospective study. J Surg Oncol. 2014;110:270-4.

7. Rueth NM, Black DM, Limmer AR, Gabriel E, Huo L, Fornage $\mathrm{BD}$, et al. Breast conservation in the setting of contemporary multimodality treatment provides excellent outcomes for patients with occult primary breast cancer. Ann Surg Oncol. 2015;22:90-5.

8. Fayanju OM, Stoll CR, Fowler S, Colditz GA, Jeffe DB, Margenthaler JA. Geographic and temporal trends in the management of occult primary breast cancer: a systematic review and meta-analysis. Ann Surg Oncol. 2013;20:3308-16.

9. Khandelwal AK, Garguilo GA. Therapeutic options for occult breast cancer: a survey of the American Society of Breast Surgeons and review of the literature. Am J Surg. 2005;190: 609-13.

10. Sagara Y, Freedman RA, Vaz-Luis I, Mallory MA, Wong SM, Aydogan F, et al. Patient prognostic score and associations with survival improvement offered by radiotherapy after breast-conserving surgery for ductal carcinoma in situ: a population-based longitudinal cohort study. J Clin Oncol. 2016;34: 1190-6.

11. Orel SG, Weinstein SP, Schnall MD, Reynolds CA, Schuchter LM, Fraker DL, et al. Breast MR imaging in patients with axillary node metastases and unknown primary malignancy. Radiology. 1999;212:543-9.

12. Vlastos G, Jean ME, Mirza AN, Mirza NQ, Kuerer HM, Ames FC, et al. Feasibility of breast preservation in the treatment of occult primary carcinoma presenting with axillary metastases. Ann Surg Oncol. 2001;8:425-31.

13. EBCTCG (Early Breast Cancer Trialists' Collaborative Group), McGale P, Taylor C, Correa C, Cutter D, Duane F, et al. Effect of radiotherapy after mastectomy and axillary surgery on 10-year recurrence and 20-year breast cancer mortality: metaanalysis of individual patient data for 8135 women in 22 randomised trials. Lancet. 2014;383:2127-35.

14. Recht A, Comen EA, Fine RE, Fleming GF, Hardenbergh PH, Ho AY, et al. Postmastectomy radiotherapy: an American Society of Clinical Oncology, American Society for Radiation Oncology, and Society of Surgical Oncology focused guideline update. Pract Radiat Oncol. 2016;6:e219-34.

15. Park HJ, Shin KH, Kim JH, Ahn SD, Kim JY, Park W, et al. Incorporating risk factors to identify the indication of postmastectomy radiotherapy in N1 breast cancer treated with optimal systemic therapy: a multicenter analysis in Korea (KROG 14-23). Cancer Res Treat. 2017;49:739-47.

16. Barton SR, Smith IE, Kirby AM, Ashley S, Walsh G, Parton M. The role of ipsilateral breast radiotherapy in management of occult primary breast cancer presenting as axillary lymphadenopathy. Eur J Cancer. 2011;47:2099-106.

17. Shannon C, Walsh G, Sapunar F, A'Hern R, Smith I. Occult primary breast carcinoma presenting as axillary lymphadenopathy. Breast. 2002;11:414-8.

18. Ellerbroek N, Holmes F, Singletary E, Evans H, Oswald M, McNeese M. Treatment of patients with isolated axillary nodal metastases from an occult primary carcinoma consistent with breast origin. Cancer. 1990;66:1461-7.

19. Blanchard DK, Farley DR. Retrospective study of women presenting with axillary metastases from occult breast carcinoma. World J Surg. 2004;28:535-9.

20. Macedo FI, Eid JJ, Flynn J, Jacobs MJ, Mittal VK. Optimal surgical management for occult breast carcinoma: a meta-analysis. Ann Surg Oncol. 2016;23:1838-44.

21. Woo SM, Son BH, Lee JW, Kim HJ, Yu JH, Ko BS, et al. Survival outcomes of different treatment methods for the ipsilateral breast of occult breast cancer patients with axillary lymph node metastasis: a single center experience. J Breast Cancer. 2013;16:410-6. 